\title{
Animating Primary Hemostasis for Medical Student Education
}

Evelyn Lockhart1,2, Michael Corrin ${ }^{1,3}$, Paula James ${ }^{4}$, Ric Lowe ${ }^{5}$, Jodie Jenkinson ${ }^{1,3}$

'Biomedical Communications, University of Toronto; 2Dept. of Pathology, University of New Mexico; ${ }^{3}$ Dept. of Biology, University of Toronto;

\section{Abstract}

Physicians have difficulty recognizing and diagnosing disorders of primary hemostasis. The root of this may lie in their education, where students are often taught hemostasis using static graphics. We aimed to create a didactic animation on primary hemostasis for medical students to be used in North American medical schools. To promote widespread use of the animation, we surveyed hemostasis educators from Canada and the US on the animation's learning objectives. The animation's script and storyboard were developed using the Animation Processing Model (APM), a psychological processing model that addresses the perceptual limitation of learners. This animation is the first biomedical animation to use the APM in its design. Furthermore, this is the first didactic hemostasis
animation which sought peer consensus for its learning objectives.

\section{Introduction}

Physicians have difficulty recognizing and diagnosing primary hemostatic disorders like von Willebrand disease.'

In medical school, hemostasis is often taught using static graphics which may fail to capture the temporal and structural complexity of the subject, leading to incorrect mental models.

As compared to static graphics, animation has been shown to improve learning outcomes

Learning outcomes are statistically higher with medical education digital tools which are developed using a specific learning theory. Furthermore, it learning objectives for the tool. ${ }^{4}$

The purpose of this project is to develop an animation for medical students which presents an introduction to primary hemostasis.

To inform the animation's content we aimed to survey educators wits

To inform the animation's content, we aimed to survey educators with
experience in teaching hemostasis in North American medical schools regarding learning objectives for the animation.

We elected to use the Animation Processing Model (APM) as the learning

The APM is a psychological processing model that addresses the perceptual

has little or no experience with the animation's content. ${ }^{5}$

\section{References}

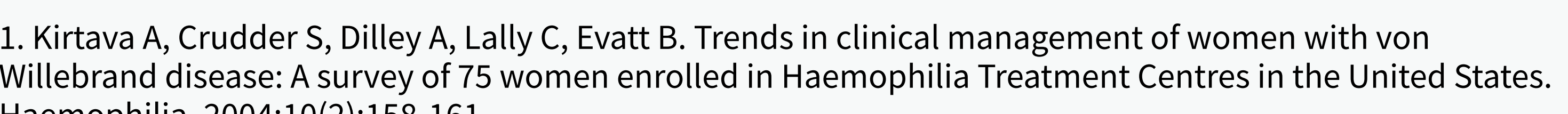
2. Lowe RK, Schnottz W. Animation Principles in Multimedia Learning. In: Mayer RE, ed. The Cambridge
Handbook of Multimedia Learning, Second Edition. Cambridge: Cambridge University Press; $2014: 513-546$

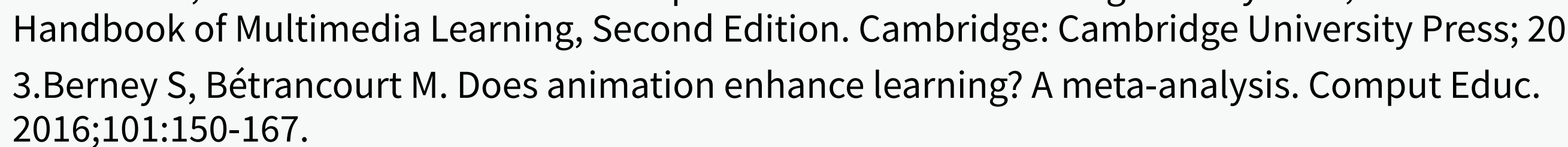

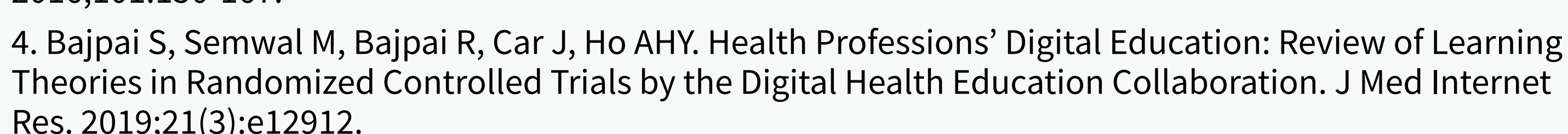

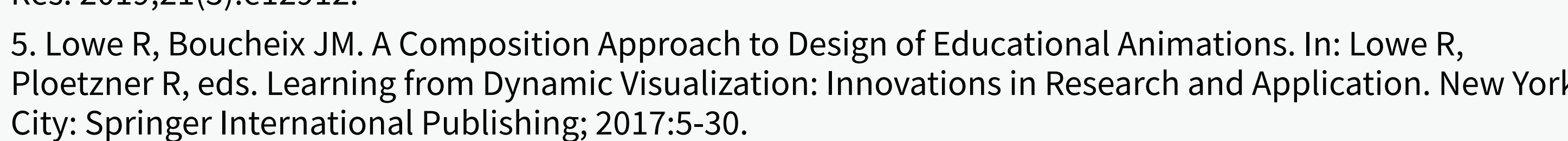

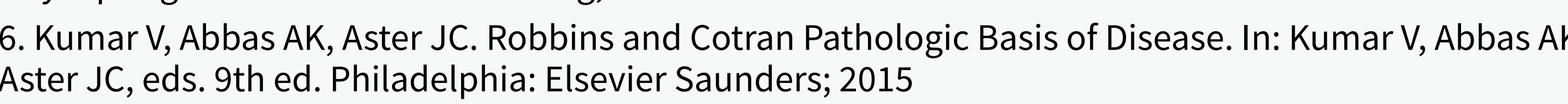

\section{Results}

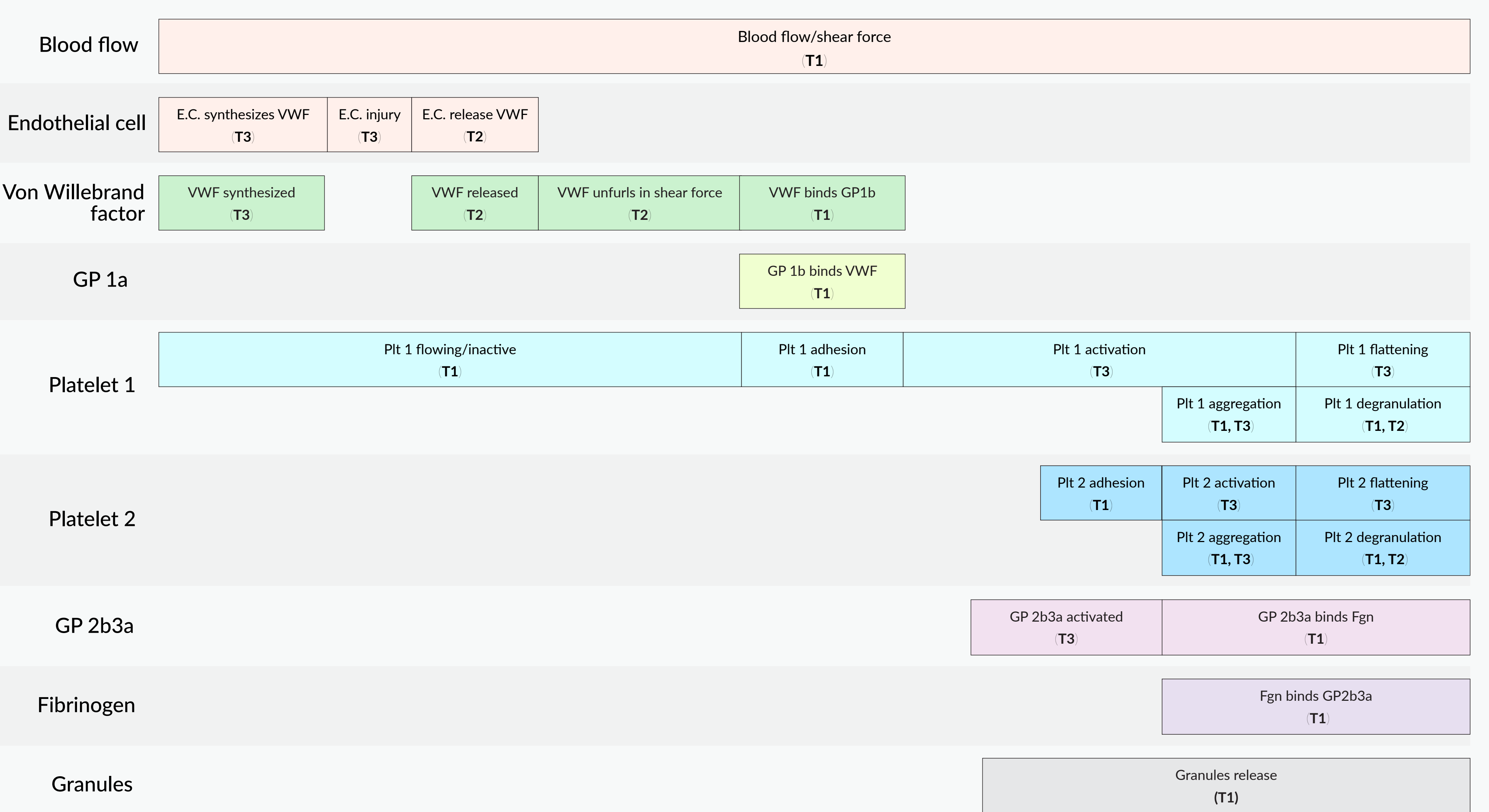

Figure 1: Event Unit Analysis of primary hemostasis learning objectives. Entity actions are indicated as follows: T1=movement, T2=appearance, T3=shape change. GP1a: glycoprotein 1a, GP 2b3a: glycoprotein
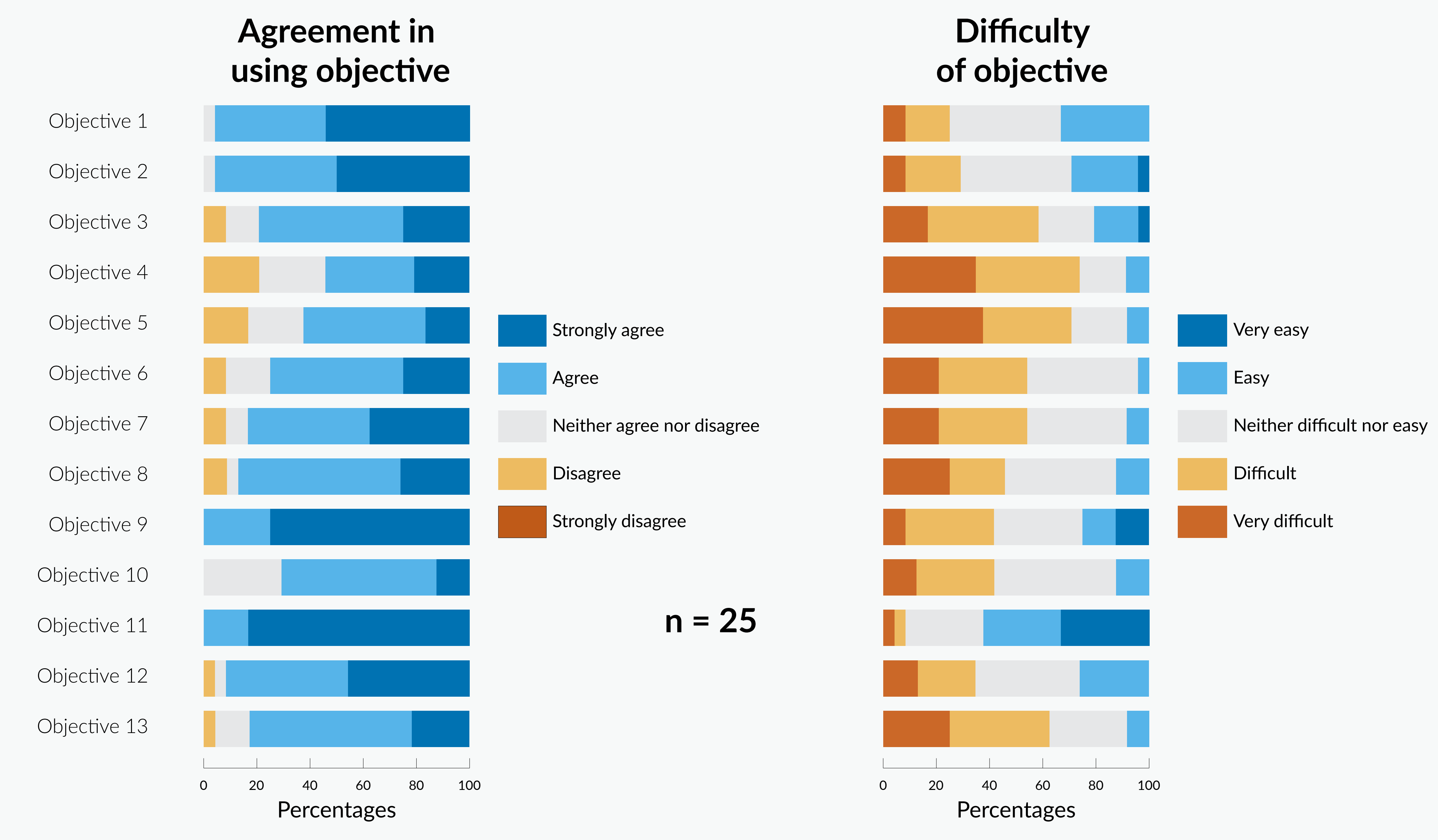

Figure 2: Summary of hemostasis educators survey on animation learning objectives.

\section{Acknowledgements}

We gratefully recognize Nicholas Woolridge (Biomedical Communications, Dept. of Biology, University of AHCDC and FWGBD for their participation in our survey and feedback on the survey instrument.

\section{Materials \& Methods}

Learning objectives were abstracted from Robbins and Cotran Pathologic Basis of Disease. 6

A Likert scale survey was sent via SurveyMonkey (www.surveymonkey.com) to (FWGBD; www.fwgbd.org).

For each proposed learning objective, the survey participant was asked: 1. The degree which the educator supported using each learning objective for the animation.

2. The difficulty faced by a typical medical student in learning the concept posed by the objective.

Objectives underwent an event unit analysis according to the APM, pairing the entities of primary hemostasis with each of their discrete actions (movement, appearance, or state change). Following this analysis, a script and storyboard was developed.

The final animation was created using two techniques:

1. Frame-by-frame animation (Rough Animator and Adobe Photoshop) 2. 2D plus animation (Maxon Cinema 4D[Sketch and Toon module], Adobe After Effects, Adobe Audition, Adobe Premiere Pro)

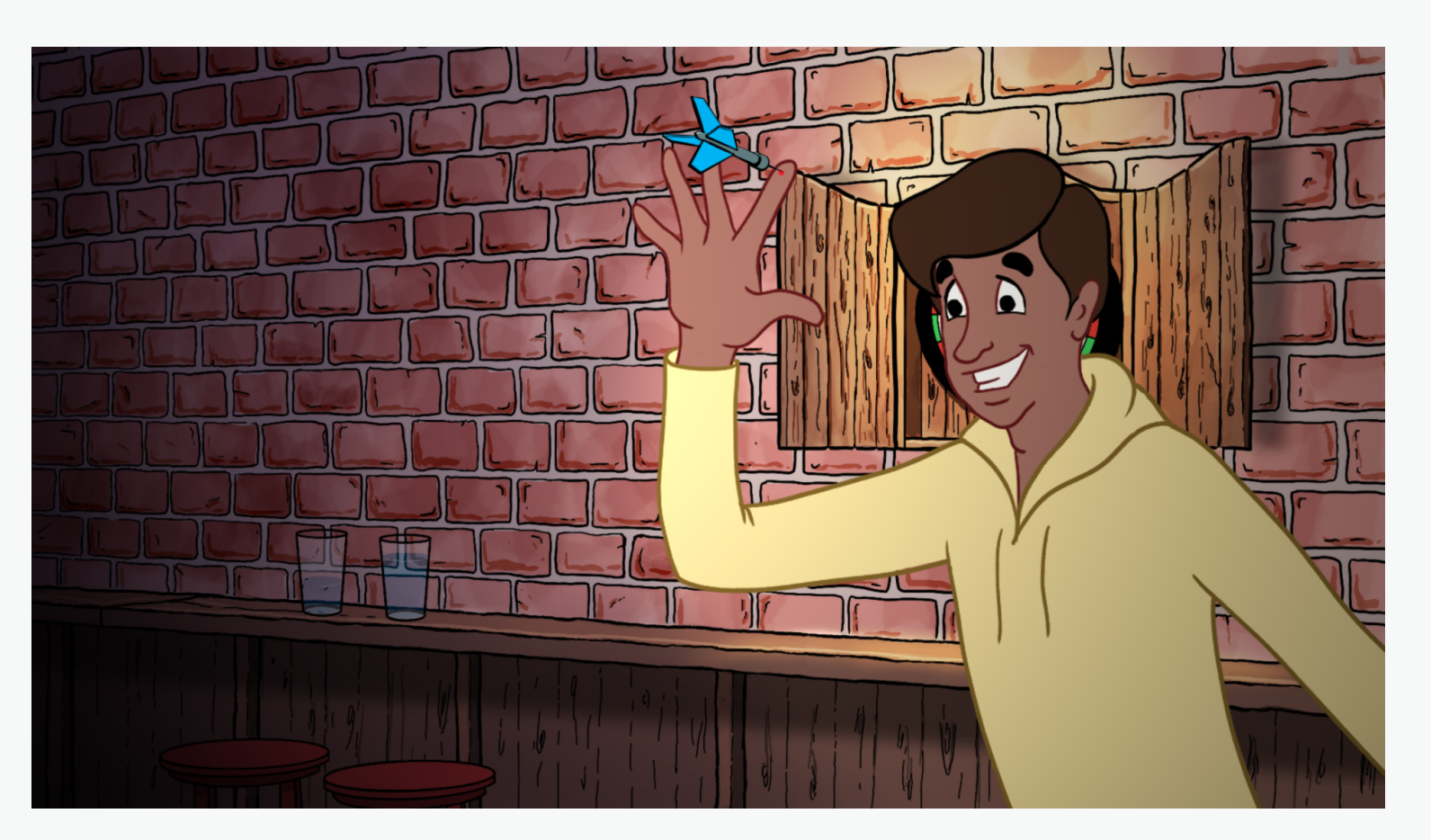

Figure 3: Frame-by-frame animation

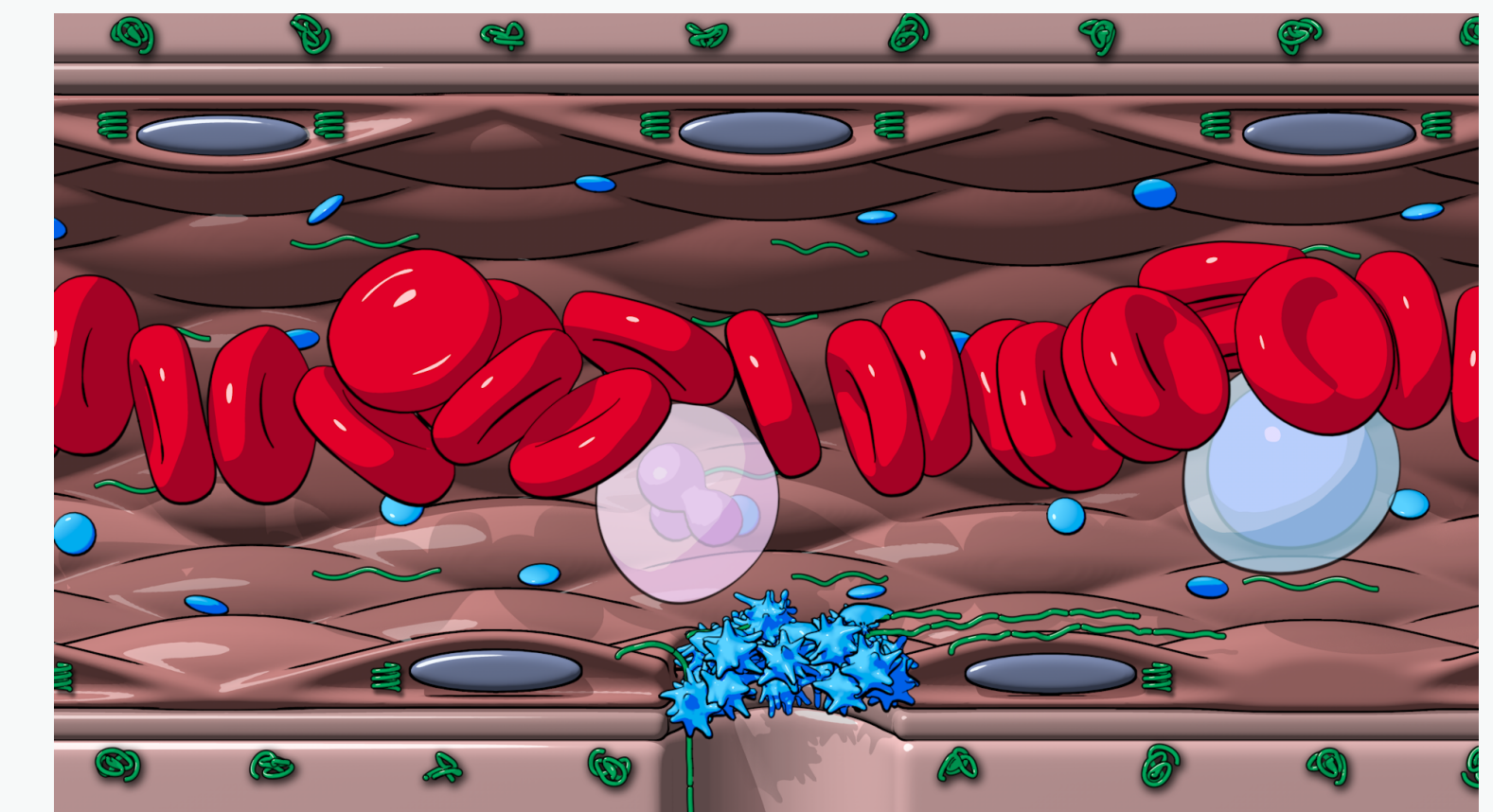

$\square$ Figure 4: $2 \mathrm{D}$ plus animation

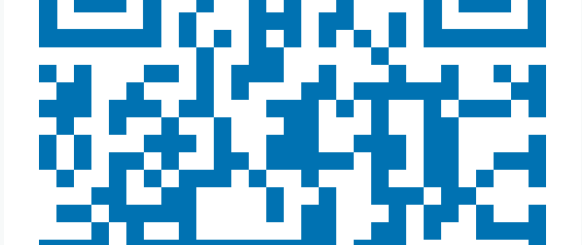

See the proposed learning objectives wwwevic, and final animation as 口ist

\section{Conclusions}

First biomedical animation to use the Animation Processing Model to inform its design.

First educational animation on primary hemostasis that sought consensus for its learning objectives.

Manuscript of results to be submitted for publication.

Future plans include:

1. Producing a control animation using a script and storyboard developed prior to the event unit analysis.

2. Performing a randomized study with medical students on learning outcomes between control and APM animations. 